\title{
$\mathrm{AD} / \mathrm{RHIC}-\mathrm{RD}-3$
}

\author{
Workshop Goals \\ (Mini-Workshop on RHIC RF Systems) \\ July 11-15, 1988 \\ Collider Center
}

H. Hahn

BNL 
MINI-WORKSHOP ON RHIC RF SYSTEM

JULY 11, 1988

REASON

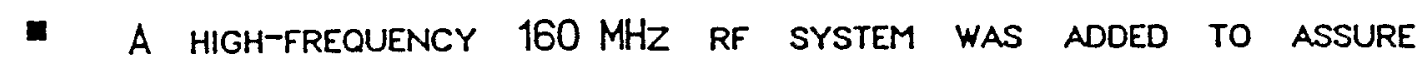
SHORT BUNCHES AND SHORT DIAMOND LENGTH DURING STORAGE MODE

- CO-EXISTENCE OF TWO RF SYSTEMS POSES NEW PROBLEMS

OBJECTIVES

- REVIEW RF SYSTEMS REQUIREMENT

DURING INJECTION, ACCELERATION, CROSSING OF TRANSITION ENERGY, TR'ANSFER FROM LOW-F TO HIGH-F SYSTEM, STORAGE OF BEAMS

- REVIEW CAVITY AND AMPLIFIER HARDWARE

VOLTAGE RANGes, NUMBER OF $160 \mathrm{MHZ}$ CAVITIES, FEEDBACK REQUIREMENTS, INTERACTION OF TWO SYSTEMS, COUPLING IMPEDANCE REQUIREMENTS, INSTABILITIES

- eStablish basis fOR REVISED CDR tEXT AND COST ESTIMATE

- WRITTEN SUMMARY AND TECH NOTES 
RELATIVISTIC HEAVY ION FACILITIES AT BNL

1986 BEGIN AGS FIXED TARGET EXPERIMENTS

ION SPECIES: $\quad{ }^{1}$ to ${ }^{28} \mathrm{~S}$ :

Beam Energy: UP to $29\left(\frac{Z}{A}\right) G E V \cdot c^{-1} / U$

FLUX: $\quad=10^{9}$ IONS/PULSE

RUNNING TIME: $\quad 5-10$ WEEKS/YEAR

1991 AGS EXPERIMENTS WITH BOOSTER SYNCHROTRON

- Extend ion mass to $A \simeq 200\left({ }^{197} \mathrm{Au}\right)$

(1909) BEGIN RHIC COLLIDER EXPERIMENTS

ION SPECIES: ${ }^{1} \mathrm{H}$ TO ${ }^{197} \mathrm{AU}$

ENERGY/BEAM: UP TO $250\left(\frac{Z}{A}\right]$ GEV/U

C.M. ENERGY: $\quad 250+250$ GEV (P) $100+100$ GEV/U (AU)

LUMINOSITY: $\quad 10^{31} \mathrm{~cm}^{-2} \mathrm{SEC}^{-1} \quad 5 \times 10^{26} \mathrm{~cm}^{-2} \mathrm{SEC}^{-1}$ 
RHIC MAJOR PARAMETERS

Energy Range (eAch beam), au

PROTONS

LUMINOSITY, AU-AU @ $100 \mathrm{GEV} / \mathrm{U}$ HEAD-ON \& $10 \mathrm{H}$ AV.

Operational lifetime au @ $>30 \mathrm{GeV} / \mathrm{U}$

DiAMOND LeNGTH @ $100 \mathrm{GEV} / \mathrm{C}$ MRAD

CiRCUMfERENCE, $4-3 / 4 \mathrm{C}_{\text {AGS }}$

NUMBER OF CROSSING POINTS

FREE SPACE AT CROSSING POINT

BETA @ CROSSING, HORIZONTAL/VERTICAL.

LOW-BETA INSERTION

BETATRON TUNE, HORIZONTAL/VERTICAL

TRANSITION ENERGY, $\gamma_{T}$

FILLING MODE

No. OF BUNCHES/RING

No. OF AU-IONS/BUNCH

FILLING TIME (EACH RING)

MAGNetic Rigidity, Bp: @ INJECTION

@ TOP ENERGY

No. OF DIPOLES (180/RING +12 COMMON)

No. OF QUADRUPOLES (276 ARC + 216 INSERTION)

Dipole Field @ $100 \mathrm{GEV} / \mathrm{U}, \mathrm{AU}$

Dipole MAGNeTIC LENGTH

DIPOLE YOKE LENGTH

COIL I.D. ARC MAGNETS

BEAM SEPARATION IN ARCS

RF FREQUENCY

RF VOLTAGE

ACCELERATION TIME

Jh711885
(7) $10.7-100 \mathrm{GEV} / \mathrm{U}$

28-250 ${ }^{+} \mathrm{GEV}$

$4.4 \times 10^{26} \mathrm{CM}^{-2} \mathrm{SEC}^{-1}$

$>10 \quad \mathrm{H}$

$\pm 27 \mathrm{cmRMS} \pm 20$

$3833.845 M$

6

$\pm 9 M$

$6 M$

$3 M$

28.82

24.8

Box-CAR

57

$1.1 \times 10^{9}$

$\sim 1$ MIN

$96.74 \mathrm{~T} \cdot \mathrm{M}$

$839.5 \mathrm{~T} \cdot \mathrm{M}$

372

492

$3.45 T$

$9.46 \mathrm{M}$

$9.7 M$

$8 \mathrm{~cm}$

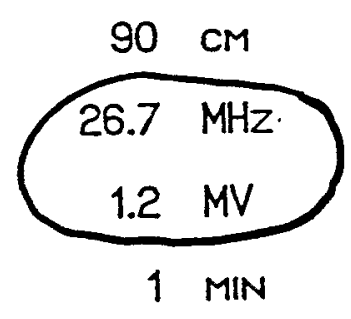




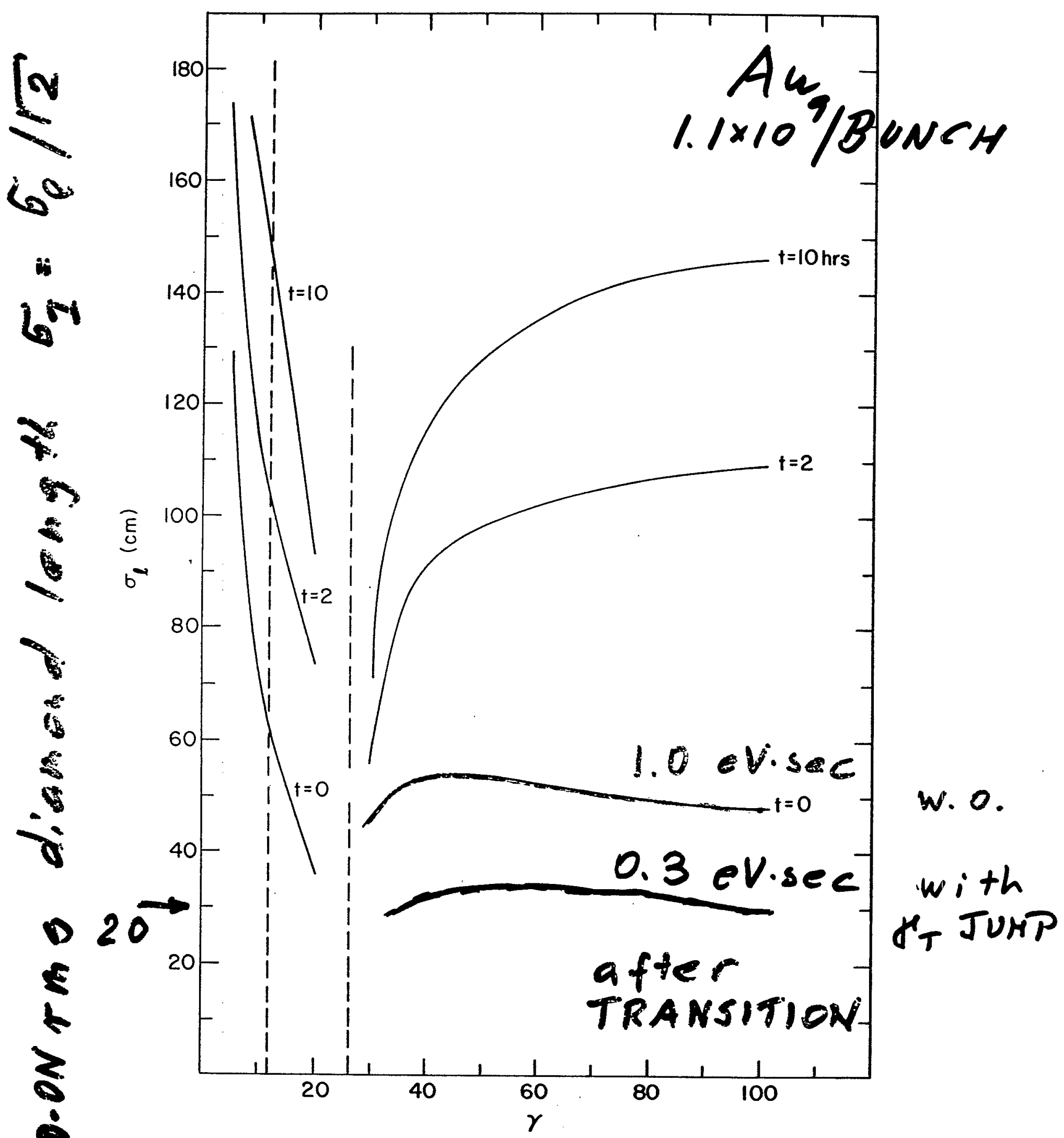




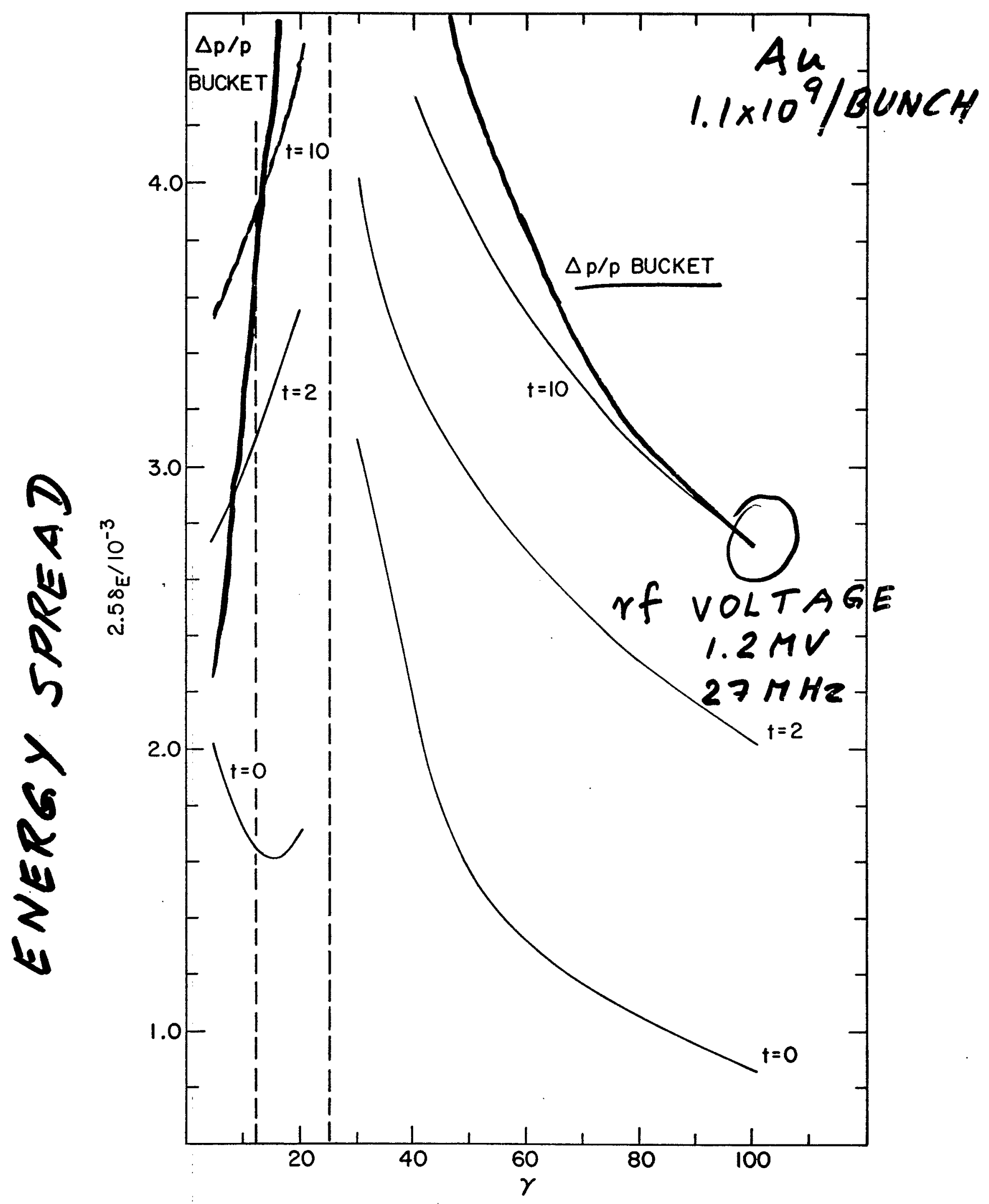




$$
\tau_{E}^{-1}=\frac{1}{\delta_{E}} \frac{\mathrm{d} \delta_{E}}{d t}=\left[\frac{\left\langle\sigma_{H}\right\rangle}{\left\langle X_{p}\right\rangle \delta_{E}}\right]^{2} \tau_{H}^{-1}
$$

with

$$
\left.\tau_{H}^{-1}=\frac{27 \pi}{2} L_{g} r_{p}^{2} E_{0} \frac{N_{B}}{S \varepsilon_{H} \varepsilon_{V}} \frac{\left\langle X_{p}\right\rangle}{\langle\beta\rangle} \frac{1}{\left(1+\left[\frac{\left\langle\sigma_{H}\right\rangle}{\left\langle X_{p}\right\rangle \delta_{E}}\right]^{2}\right.}\right]^{1 / 2}\left(\frac{Q^{2}}{A}\right]^{2}
$$

where

$$
\begin{aligned}
L_{g} & \approx 20 \\
r_{P} & =\frac{\mu_{0} e^{2} c^{2}}{4 \pi E_{O}} \\
\left\langle\sigma_{H}\right\rangle & =\left[\frac{\varepsilon_{H}}{6 \pi} \frac{\langle\beta\rangle}{\gamma}\right]^{1 / 2} \\
S & =6 \pi \sigma_{\ell} \delta_{E} \gamma E_{0} / C \\
\varepsilon_{H, V} & =\text { normalized transverse emittance } \\
\left\langle X{ }_{P}\right\rangle & =\text { averaged dispersion } \\
\langle\beta\rangle & =\text { averaged betatron function }
\end{aligned}
$$

G. Parzen, Nucl. Instr. Meth. A251, p. 220 (1986), A256, p. 231 (1987). 


\section{DIAMOND LENGTH}

REQUIREMENT

$$
\sigma_{I} \leq 20 \mathrm{CM} \text { RMS }
$$

BUNCH RMS LENGTH

$$
\sigma_{\ell}=\sqrt{2} \quad \sigma_{I} \approx 28 \mathrm{cM} \text { RMS }
$$

BUCKET HALF LENGTH

$$
160 \mathrm{MHz}(\mathrm{h}=2052)
$$

$$
\ell_{B}=\pi R / h=0.934 \mathrm{M}
$$

DUE TO BUCKET NONLINEARITY $\sigma_{\ell}<l_{B} / \sqrt{6}=38 \mathrm{~cm}$ RMS

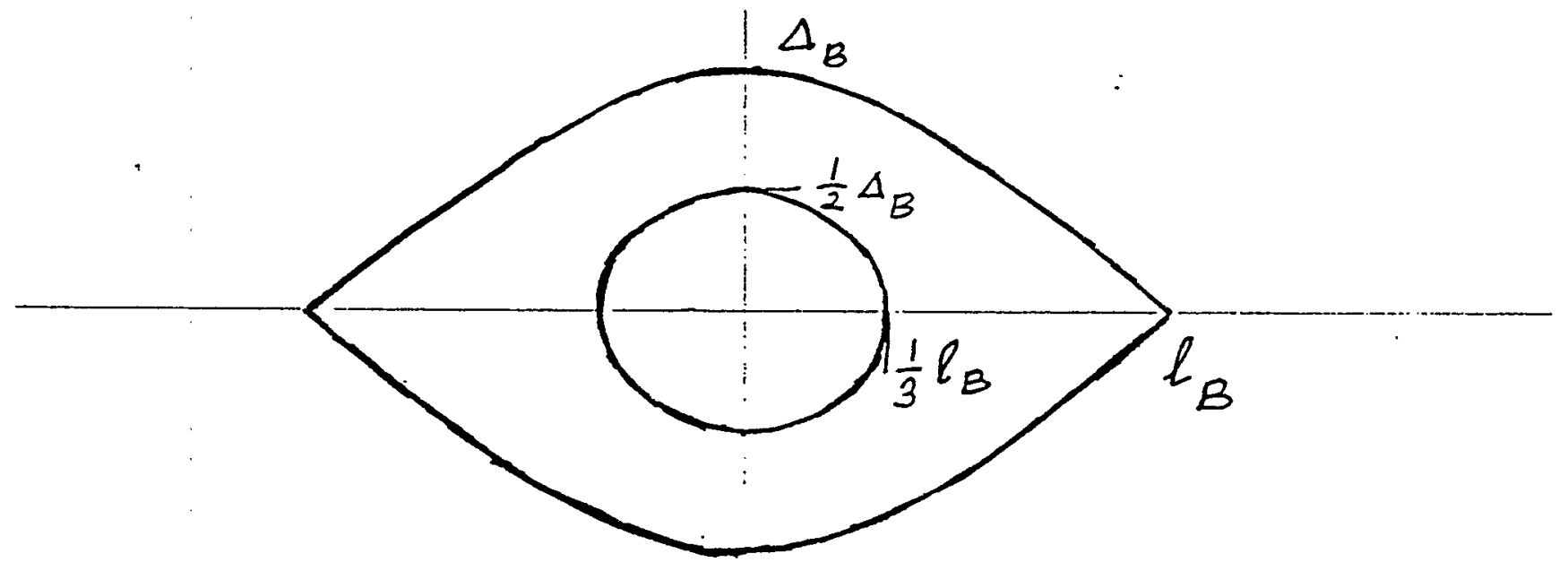

$$
\sigma_{E}=\Delta_{B} / \sqrt{6} \text { YIELDS } \sigma_{\ell}=0.268 \times l_{B}=25 \mathrm{cM} \text { RMS }
$$

ECONOMY CONDITION:

$$
\sigma_{E}=\Delta_{B} / 2
$$

$$
\sigma_{\ell}=l_{B} / 3=31 \mathrm{cM} \text { RMS }
$$




\section{RF BUCKET AND LONGITUDINAL BUNCH PARAMETERS}

BUCKET HALF HEIGHT

$$
\Delta_{B}=\left[\frac{2 \cdot \mathrm{e} \mathrm{V}}{\pi \mathrm{h}|\eta| \gamma E_{\mathrm{o}}} \frac{\mathrm{Q}}{\mathrm{A}}\right]^{1 / 2}
$$

BUCKET AREA AMU

$$
A_{B}=8 \Delta_{B} \frac{\gamma E_{O}}{h \omega_{O}}
$$

BUNCH PHASE HALF WIDTH $0<\phi<\pi$

COLE-MORTON:

$$
N=\sin ^{2} \phi / 2
$$

RMS BUNCH LENGTH

$$
\sigma_{\ell}=\frac{1}{\sqrt{6}} \quad \frac{R}{h} \phi
$$

RMS BUNCH HEIGHT

$$
\delta_{E}=\frac{1}{\sqrt{6}} \Delta_{B} \sin \phi / 2
$$

BUNCH AREA/AMU

$$
\begin{aligned}
S & =A_{B}\{(N-1) \mathrm{K}(\mathrm{N})+E(N)\} \\
\vdots & \approx 6 \pi \sigma_{\ell} \delta_{E} \gamma E_{0} / c
\end{aligned}
$$




\section{RHIC RF SYSTEMS PARAMETER}

- Beam parameters from agS

$$
\left.\begin{array}{ll}
\text { p } & 10^{11} / \mathrm{BUNCH} \\
\text { AU } 10^{9} / \mathrm{BUNCH}
\end{array}\right\} \begin{aligned}
& 0.3 \mathrm{EV} \cdot \mathrm{SEC} / \mathrm{U} \\
& 10 \pi \mathrm{MM} \cdot \mathrm{MRAD}
\end{aligned}
$$

- Assume essentially no growth of BUNCH aREa at inJection and DURING TRANSITION

ACCELERATION RF SYSTEM

2 CAVITIES (CDR-TYPE)

TUNING RANGE

$$
\begin{aligned}
& \text { STORAGE RF SYSTEM } \\
& \text { TUNING RANGE } \\
& \text { VOLTAGE @ } 10 \mathrm{HR}
\end{aligned}
$$

$26.7 \mathrm{MHz}$

$300 \mathrm{kV}$

1\% (> $7 \mathrm{GEV} / \mathrm{U})$

$$
\begin{aligned}
& 160 \mathrm{MHz} \\
& 0.1 \%-(>30 \mathrm{GEV} / U) \\
& 11.4 \mathrm{MV}-\mathrm{AU}\left(\Delta_{B}=2 \sigma_{E}\right) \\
& 17.7 \mathrm{MV}-\mathrm{AU}\left(\Delta_{B}=\sqrt{6} \sigma_{E}\right) \\
& 2.3 \mathrm{MV}-\mathrm{P}\left(\Delta_{B}=\sqrt{6} \sigma_{E}\right)
\end{aligned}
$$

SCALED CERN-TYPE CAVITY

NUMBER OF CAVITIES, VOLTAGE/CAVITY ?

SUPERCONDUCTING CAVITIES ?

- DEsign for future UPGRADE

$2 \times 57$ BUNCHES, $2 \times$ NUMBER IONS/BUNCH

- DESIGN FOR 2 - 3 MISSING BUNChES (BEAM DUMP GAP ) Jh71188d 


\section{MINI WORKSHOP ON RHIC RF SYSTEMS \\ Monday, July 11 -- 2:30 PM \\ BLG. 1005 Conference RoOM 4TH Floor}

WELCOME \& WORKSHOP GOALS

H. HAHN

RF SYSTEMS OVERVIEW

A.G. RuggIERo ,

INTRABEAM SCATTERING ResUltS

G. PARzen

Beam TRANSFER AGS/RHIC,

LOW F - HIGH F

E.C. RAKA

Passage through Transition

S.Y. LEE

STMULATION OF TRANSITION

\& TRANSFER

J. WEI

RF CAVITIES AND AMpLtFIERS

J.G. Cottingham

Gamma-Transition Quadrupoles

P.A. THOM PSON 